\title{
PIV measurements on the formation of the flow field and aerosol particle distribution in a turbulent square duct flow
}

\author{
T. Barth, M. Banowski \& U. Hampel \\ Institute of Safety Research, \\ Helmholtz-Zentrum Dresden-Rossendorf e.V., Germany
}

\begin{abstract}
The formation of the flow field and spatial aerosol particle distribution play an important role in various industrial applications. Regarding experiments on aerosol particle transport phenomena in turbulent flows it is of essential interest to generate a homogeneously mixed air stream. Particle Image Velocimetry (PIV) measurements have been conducted in order to characterize the turbulent mixing process in a small-scale gas/aerosol test facility which is designed for the investigation of deposition and resuspension of nuclear aerosol particles. The turbulent flow field in a square duct $\left(R e_{d}=8.9 k .43 k\right)$ is seeded with DEHS droplets in the size range of approximately $d_{\text {Paero }}=2 \mu \mathrm{m}$. Firstly, the downstream formation of particle mixing is quantified in terms of the spatial distribution of the scatter light intensity of the PIV images. It is found that sufficient mixing of the aerosol particles is accomplished at 8 to 10 hydraulic diameters downstream of the injection point. Furthermore, the formation of the flow field is evaluated in terms of the time mean averaged velocity as well as velocity fluctuations. These values are presented at successive downstream positions and display the development of the turbulent boundary layer. It is shown that the near wall turbulent region of the test section nicely follows the universal slope of a well developed turbulent boundary layer. Nevertheless, the flow field formation still takes place after 20 hydraulic diameters.
\end{abstract}

Keywords: aerosol particles, particle image velocimetry, turbulent flow, quadrant count method. 


\section{Introduction}

The nature of turbulent flows in stacks and ducts has been comprehensively explored in the past by all kinds of scientists but is not yet fully understood. Huser and Biringen [4] described the nature of turbulent duct flow as a mean flow, which is superimposed by eight counter rotating edge vortices. They performed a direct numerical simulation at a bulk Reynolds number of 10320 , which displays the interaction between corner near-wall effects and the distortion of the mean flow field. Quadrant analysis of the instantaneous turbulent structures shows a dependency between the stress-driven secondary flow field with respect to pressure-strain and velocity-pressure gradient correlations.

Another direct numerical simulation of a turbulent duct flow at Reynolds number of 6000 was done by Nikitin [5] who focussed on the characterization of near-wall turbulence statistics. Comparison of the time mean averaged flow field between Nikitin's data and present results show relatively good agreement.

Recently, Winkler et al. [10] performed a Large Eddy Simulation (LES) of a turbulent square duct flow at Reynolds numbers of 360 based on the friction velocity. It is shown that aerosol particles tend to accumulate in regions of low vorticity magnitude. Nevertheless, the time mean averaged flow field is in good agreement to the present results with respect to the state of flow formation.

In this work, Particle Image Velocimetry (PIV) measurements have been conducted in a new small-scale gas/aerosol test facility whose results will be presented hereafter. For experimental studies, it is of essential importance to understand how well developed the flow field in the test section of a facility is. Unknown flow disturbances due to flow formation processes may have a crucial impact on the quality of the experimental results. Thus, the state of flow development and particle mixing in the present facility are quantified experimentally. The experiments have been conducted in an air driven wind tunnel, which is specifically designed for the investigation of the transport behaviour of nuclear aerosol particles. The flow field is recorded successively at different downstream positions and Reynolds numbers. Analysis of scattered light intensity distribution of the PIV raw data images by means of quadrant count techniques characterizes the particle mixing process. It is shown that the aerosol particles are homogeneously distributed 8 hydraulic diameters downstream of the injection point which is in good agreement to published data (Cohen [2]). The flow field formation is illustrated in terms of time averaged velocity plots in channel centre section. It will be shown that the velocity profiles nicely approach existing numerical data approximately after 40 to 50 hydraulic diameters. However, the turbulent boundary layer is in excellent agreement to results from direct numerical simulations.

\section{Experimental setup}

The facility is an air driven open loop wind tunnel in suction mode. The inlet of the tunnel is equipped with a HEPA for air cleaning purposes. Afterwards, a nozzle contracts the flow into a $10 \times 10 \mathrm{~cm}^{2}$ square duct formation zone of 15 
diameters length. The test section is located downstream of the flow formation zone and has a length of 5 hydraulic diameters. Both the formation zone and test section are made of Plexiglas in order to allow optical access. Finally, the particle laden air stream is decelerated in a diffuser stage and filtered by an electrostatic precipitator before entering a radial fan at the outlet of the facility.

Aerosol particles, which are used as PIV seeding, are made of DEHS by means of a Condensation Aerosol Generator SLG 270 and injected into the flow upstream of the flow formation zone. The aerodynamic diameter of the aerosol particles was measured using an Aerodynamic Particle Sizer Spectrometer (TSI, APS 3321) and isokinetic sampling $\left(d_{P, \text { aero }}=2 \mu \mathrm{m}\right)$.

The LaVision 2C2D-PIV system consists of a Nd:YAG double pulse laser source (New Wave Gemini 200-15) and a CCD camera (Imager intense, $1376 x$ $1040 \mathrm{pxl}$ ) focusing normal to the laser sheet as further described in Raffel et al. [8]. Both, the laser and the camera are mounted on a 3D linear traversing system in order to record the flow field at different downstream positions using the same calibration. The double frame, double exposure technique is used and the images have been processed with LaVision $\mathrm{DaVis} 7$.

The signal-to-noise ratio of the images is increased substantially by calculating the mean pixel-image of a data set and then subtracting it from every individual image. This eliminates background noise, e.g., due to scatter light reflections. The images were then cross-correlated. As correlating attributes were chosen an interrogation window size of $32 \times 32$ pixels at positions $x=[1,3,5] d$ and $16 \times 16$ pixels further downstream due to more homogeneous mixing of the tracer particles. The images were correlated with $75 \%$ overlap. Thereby the spatial resolution of the velocity field was about 0.4 down to $0.2 \mathrm{~mm}$ per interrogation frame. According to Prasad et al. [7], the relative uncertainty of the velocity is about $4 \%$ of the measured value. 400 sections were recorded and time averaged to have sufficient statistical convergence of the turbulent flow field.
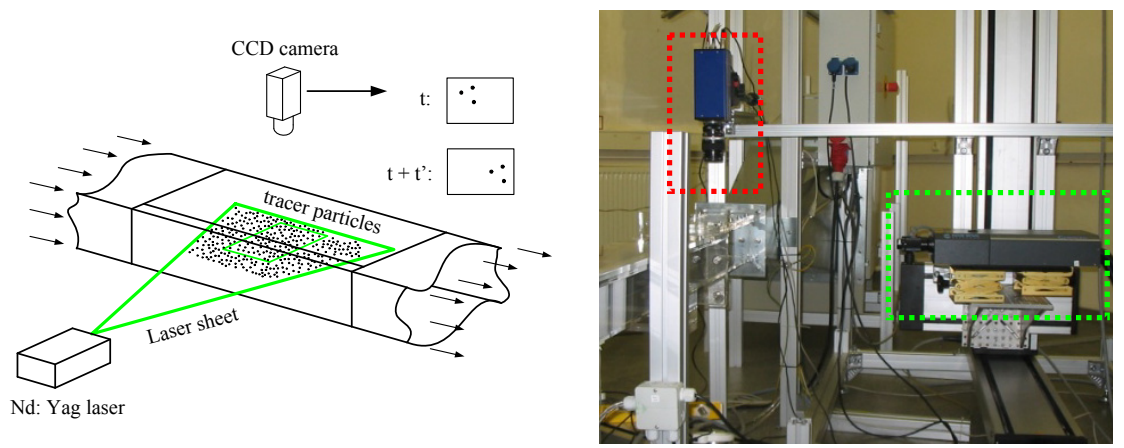

Figure 1: Left: general setup of 2C2D PIV system (Raffel et al. [8]), right: test section including Nd:YAG laser (green) and CCD camera (red). 


\section{Results}

\subsection{Spatial distribution of aerosol particle mixing}

The quality of the PIV data is mainly influenced by an evenly distributed tracer material, which is illuminated by two well aligned laser sheets. At the entrance to the flow formation zone, the tracer particles are irregularly distributed, as can be seen in Figure 2. PIV raw image data display the kind of vortex shedding in the wake of the particle injection nozzle. Particles tend to accumulate in the vicinity of the vortices. Eventually the turbulent mixing leads to a homogeneous spatial distribution of the tracer particles in the downstream region. This process is quantified by spatial point pattern analysis using quadrant count method (Diggle [3]).
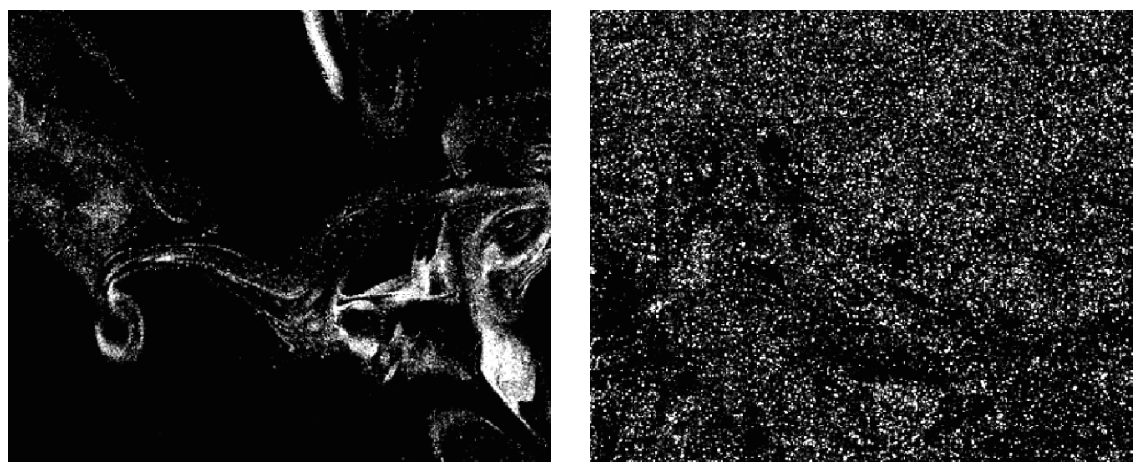

Figure 2: $\quad$ PIV raw data images, left: $x=1 d$, right: $x=17 d$.

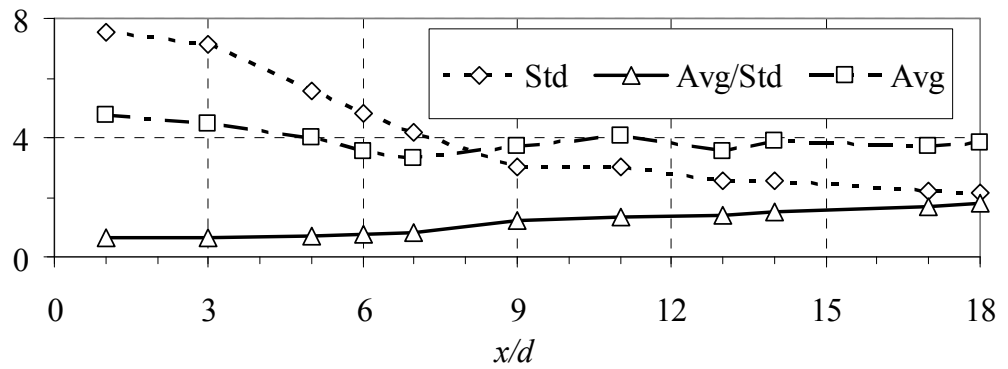

Figure 3: Downstream formation of particle distribution and concentration in terms of scatter light intensity.

The PIV raw images are post processed by means of Image J. 50 snapshots are uploaded and discretized into $40 \times 32$ interrogation windows. Then, the stack of 50 images is treated with a threshold to suppress optical noise and mean average of particle light intensity $(A v g)$, as well its standard deviation $(S t d)$ are evaluated. The standard deviation of particle light intensity decreases 
continuously from 7.5 down to 2.13 which is probably the result of continuous turbulent mixing downstream.

The average light intensity between inlet and test section decreases slightly to about $20 \%$ which might be induced by the deposition of the tracer particles at the channel walls. Assuming that there is constant light sheet intensity, the downstream gradient average light intensity might be a parameter, which could be used in order to quantify particle deposition rates. Nevertheless, normalizing the mean average particle light intensity $(A v g)$ by standard deviation $(S t d)$ leads to a particle number independent value for the correct assessment of spatial distribution. The ratio $\mathrm{Avg} / \mathrm{Std}$ increases from $9 \%$ at $x / d=1$ to about $49 \%$, but the uptake from $x / d=9$ to 18 reduces to $31 \%$. According to that change, the tracer particles are roughly well distributed after 8 hydraulic diameters, but there is still progress in the mixing process due to particle deposition and turbulent mixing. However, the aerosol particles seem to be evenly distributed 8 hydraulic diameters downstream of the injection point, which is in good agreement with the empirical values of Cohen [2] who also stated that homogeneously mixing is evident after 5 to 8 hydraulic diameters downstream of a disturbance.

\subsection{Formation of the velocity field}

The development of the flow field is illustrated in terms of non-dimensional velocity plots across the channel centre plane at different downstream positions. The time-averaged mean velocities, as well as its fluctuations are computed from 400 instantaneous velocity fields (Figure 4).

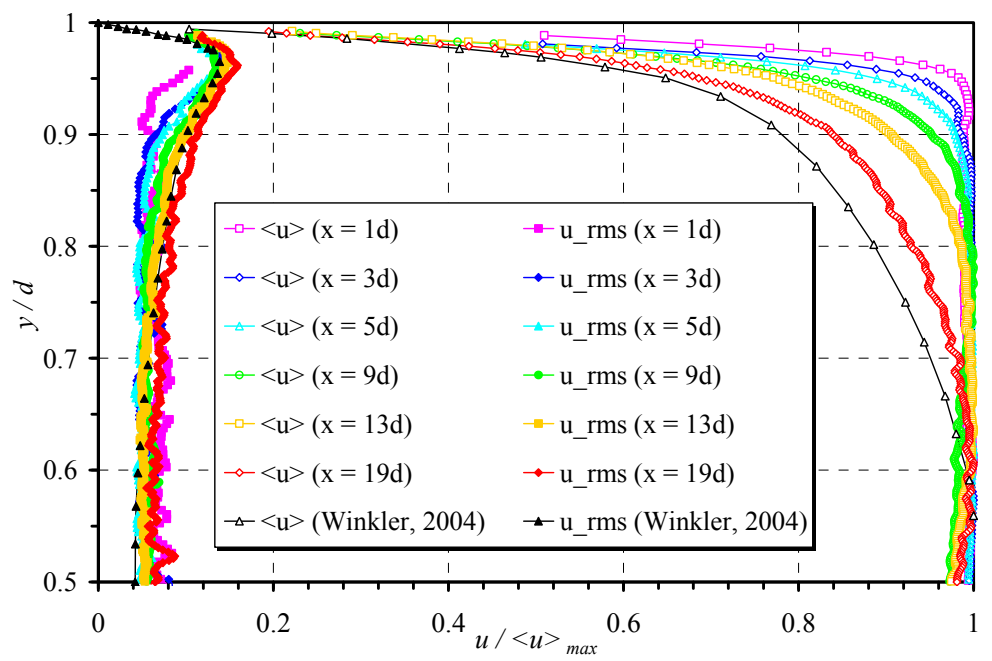

Figure 4: $\quad$ Time mean averaged velocity profiles and root mean square values across channel centre plane at $R e_{d}=8.9 \mathrm{k}$.

The velocity profile at $x=1 d$ (magenta line) is considerably sharp, whereas the slope with increasing downstream position continuously changes to a well- 
curved profile. Note that the development of the flow has not yet been completed at $x=19 d$ (red line).

There is a slight shift of $\langle u\rangle_{\max }$ to $y / d=0.6$, but $\langle u\rangle_{\max }$ should usually be in channel centre. One possible reason for this is the asymmetric injection of the tracer particles in order to concentrate the tracer particles in the measurement area. The SLG 270 is driven by a $\mathrm{N}_{2}$ gas flux of about $d V / d t=150 \mathrm{l} / \mathrm{h}$, which is a contribution to the overall volume flux in the test section of approximately $1 \%$. However, the time-averaged mean velocity profiles seem to approach the slope of LES data published by Winkler et al. [10]. It is assumed that this approach might be approximately finished after 20 hydraulic diameters further downstream.

The profiles of the root mean square velocities in Figure 4 match the data of Winkler et al. [10]. The slope of $u_{r m s}$ corresponds to the slope of Winkler's data at a downstream position of $x=3 d$. The maximum of $u_{r m s}(x=3 d)$ in the nearwall region is nicely reproduced. Further downstream the magnitude of $u_{r m s}$ overshoots the level of Winkler (Winkler et al. [10]), which might be an effect of higher wall roughness and surface irregularities of the experimental facility.

The velocity fluctuations could not be resolved in the viscous sublayer of the wall-near region due to reflexions and laser sheet distortion at the wall.

Furthermore, the non-dimensional, time-averaged mean velocity $u^{+}$is plotted against the wall units $y^{+}$in Figure 5 in order to compare the data with the universal slope of a turbulent boundary layer (tbl). The dotted line represents the ideal tbl, whereas in the viscous sublayer $\left(y^{+}<5\right)$ the velocity is proportional to the wall units $\left(u^{+}=y^{+}\right)$. For $y^{+}>12$ the velocity $u^{+}$departs from the linear relationship and above $y^{+}>20$ it enters the logarithmic law of the wall $\left(u^{+}=1 / \kappa\right.$ $\ln \left(y^{+}\right)+B$ ), which is called the log-law region. The intermediate region between viscous sublayer and log-law region is called buffer layer (Pope [6]).

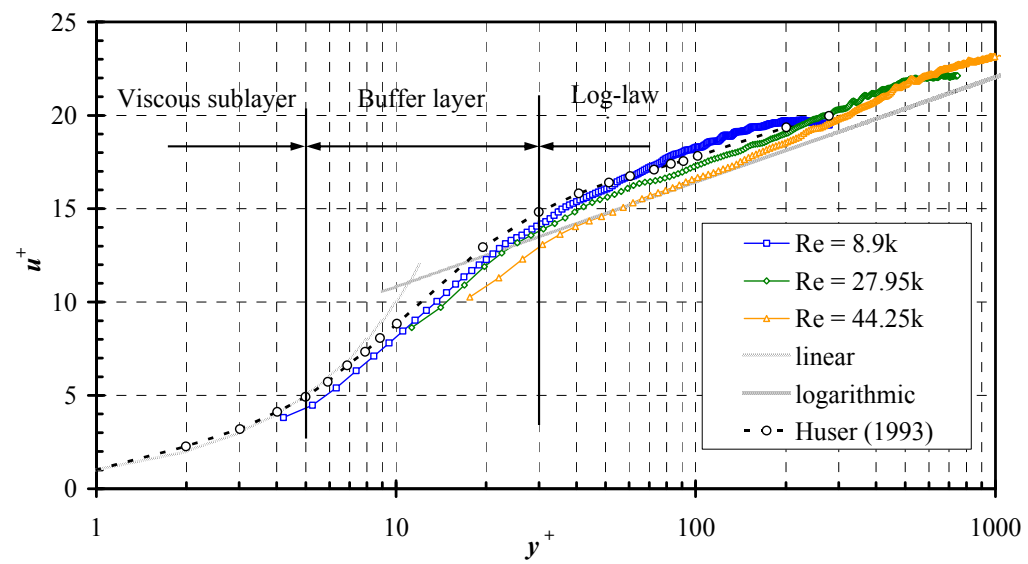

Figure 5: Time mean averaged dimensionless velocity $u^{+}$against wall units $y^{+}$in channel centre plane at $x=17 d$. 
The first measurement point of the low Reynolds number $R e_{d}=8.9 \mathrm{k}$ is at $y^{+}=4$ which is fairly well within the viscous sublayer. In comparison to the law of the wall (hatched gray line) the measured velocity falls below the $u^{+}=y^{+}$ slope in the viscous sublayer and slightly exceeds the log law above $y^{+}>20$. This might be related to the unfinished development of the turbulent flow field at this position downstream of the inlet. Note that by increasing the Reynolds number from $R e_{d}=8.9 k$ to $R e_{d}=27.95 k$ and finally $R e_{d}=44.25 k$ the first useable measurement point changes from $y^{+}=4$ to $y^{+}=11$ and finally to $y^{+}>18$. By increasing the Reynolds number, the viscous length scale decreases and thus the layers of the tbl shrinks by then. Therefore, the application of a constant geometrical measurement grid defined by the settings of the PIV system leads to the spatial resolution of the tbl to decrease with increasing Reynolds number. However, the data from Huser and Biringen [4] show excellent agreement to the present data and also overshoot the log-law region at $y^{+}>20$. They state that the overshooting in the log-law region might be due to the higher turbulence production near the walls away from the corners compared to channel and boundary layer flows without pressure gradient.

\section{Discussion}

The time-averaged mean velocity plots in channel centre (Figure 4 and Figure 5 ) show reasonably good agreement with the numerical results published by Winkler et al. [10] and Huser and Biringen [4]. Note the non dimensional velocity in channel centre is proportional to Reynolds number. This relationship is consistent to the numerical results of Ciofalo and Collins [1], which is quantified in Table 1. In contrast, the time-averaged mean fluctuations in channel centre are assumed to be $u^{+}=1.4$ by Nikitin [5] and $u^{+}=2.2$ by Truckenbrodt [9]. In the present work the time-averaged mean fluctuations increase from the inlet $u^{+}(x / d=1)=0.85$ up to $u^{+,}(x / d=17)=2.45$ in the test section, which suits well with the value from Truckenbrodt [9]. The increase in the magnitude of the fluctuations is assumed to be an indication of an increasing turbulence in the downstream region.

Table 1: Comparison of non dimensional maximal velocity against Reynolds number.

\begin{tabular}{cccc}
\hline \multicolumn{2}{c}{ present study } & \multicolumn{2}{c}{ Ciofalo and Collins [1] } \\
\hline $\operatorname{Re}(k)$ & $u_{\max }^{+}(-)$ & $\operatorname{Re}(k)$ & $u_{\max }^{+}(-)$ \\
\hline 8.9 & 19.77 & 10 & 20.5 \\
27.95 & 21.92 & 20 & 21 \\
44.25 & 22.68 & 40 & 22 \\
\hline
\end{tabular}

Considering the time-averaged mean flow field presented in Figure 4 and Figure 5 , it is visible that in terms of downstream length the near-wall turbulent boundary layer develops much faster than the core region. The root mean square 
of the mean velocity already meets the curve progression of Winkler et al. [10] after 3 hydraulic diameters downstream of the inlet whereas the slope of the time mean averaged velocity still changes after 20 hydraulic diameters.

\section{Conclusion}

In the present work a PIV measurement of a turbulent flow in a square duct was done in order to assess the aerosol particle mixing process and the flow development in terms of velocity profiles at the channel centre. At first, a brief overview on published data about turbulent square duct flow was given. Following the remarks of Huser and Biringen [4] a turbulent square duct flow consists of eight counter-rotating edge vortices which deform the mean flow field accordingly. The downstream particle mixing process was analyzed by quadrant count method. The distribution of the scattered light intensity of the PIV raw data images was analyzed by dividing the image into separate interrogation frames and assessing the scattered light intensity of each frame, respectively. It was shown that particle mixing is complete after 8 hydraulic diameters, which is in good agreement to previous investigations on turbulent particle mixing.

The flow field formation was illustrated in terms of time-averaged mean velocity profiles in the channel centre. The curve progression of the mean velocity right after the inlet is relatively sharp and thereafter the slope continuously approaches a parabolic shape. However, the present velocity field in the test section $(x=15 . .20 d)$ is not fully developed, but it seems to approach the flow field of Winkler et al. [10] after approximately further 20 hydraulic diameters downstream. On the other hand, the velocity root mean square already agrees well with Winkler's data after 3 hydraulic diameters, which raises the suggestion that the wall-near turbulent boundary layer develops faster than the core flow. Furthermore, this assumption was endorsed by the plot of non dimensional velocity $u^{+}$against wall units $y^{+}$. In comparison to the universal law of the wall (Pope [6]) and DNS data published by Huser and Biringen [4], there is excellent agreement between these data and the present results. In addition, the overshooting of the velocity in the log-law region can be explained by additional turbulence production in the channel corner. This turbulent production may have an impact on the channel core flow. In relying on the present data, it is assumed that the turbulent boundary layer region converges much earlier a constant state with respect to downstream formation compared to the channel core flow. In the present facility, the particle mixing is complete after 8 hydraulic diameters downstream of the injection point. The near-wall turbulent boundary layer nicely follows the universal law of the wall after 17 hydraulic diameters but the core flow probably needs 40 to 50 hydraulic diameters to approach an equilibrium state between the pressure and the shear forces. 


\section{References}

[1] Ciofalo, M. and Collins, M.W., Large-Eddy Simulation of turbulent Flow and Heat Transfer in plane and rib-roughened Channels. International Journal for numerical Methods in Fluids, 15(4), pp. 453-489, 1992.

[2] Cohen, B.S, Air Sampling Instruments for Evaluation of Atmospheric Contaminants. American Conference of Governmental and Industrial Hygenists, p. 595, 2001.

[3] Diggle, P., Statistical analysis of spatial point patterns, Arnold, 2003.

[4] Huser, A. and Biringen, S., Direct numerical simulation of turbulent flow in a square duct. Journal of Fluid Mechanics, 257, pp. 65-95, 1993.

[5] Nikitin, N.V., Statistical Characteristics of Wall Turbulence. Fluid Dynamics, 31(3), pp. 361-370, 1996.

[6] Pope, S.B., Turbulent Flows. Cambridge University Press, 2009.

[7] Prasad, A. K.,; Adrian, R. J.; Landreth, C. C. and Offutt P. W., Effect of resolution on the speed and accuracy of particle image velocimetry interrogation, Experiments in Fluids, 13, pp. 105-116, 1992.

[8] Raffel, M.; Willert, C. E.; Werely, S. T. and Kompenhans, J., Particle Image Velocimetry - A Practical Guide. Springer-Verlag: Berlin and Heidelberg, 2007.

[9] Truckenbrodt , E., Fluidmechanik Band 1 - Grundlagen und elementare Strömungsvorgänge dichtebeständiger Fluide. Springer-Verlag: Berlin and Heidelberg, 2008.

[10] Winkler, C. M.; Rani, S. L. and Vanka, S. P., Preferential concentration of particles in a fully developed turbulent square duct flow. International Journal of Multiphase Flow, 30(1), pp. 27-50, 2004. 